\title{
ON THE STABILITY OF THE SUPERSONIC FLOWS PAST A WEDGE*
}

\author{
BY \\ G. F. CARRIER \\ Brown University
}

1. Introduction. When an ideal ${ }^{1}$ compressible gas approaches a symmetrically oriented infinite wedge with uniform supersonic speed, there exist for many wedge angles and upstream velocity magnitudes two alternative steady flow configurations. Associated with one of the flow fields is a plane shock which is attached to the wedge vertex and downstream of which is a uniform subsonic flow. The alternative solution includes a "weaker" attached shock which is more sharply inclined to the wedge surface. Downstream of this shock there also exists a uniform flow field with a larger velocity than that of the strong shock solution. Usually, in this latter case, the downstream flow is supersonic but there is comparatively small range of "inlet" conditions for which it is subsonic. The weak shock is usually observed in experimental work although with sufficient care one can observe the strong shock configuration. ${ }^{2}$

It seems of interest, then, to see what a perfect fluid theory will predict in regard to the stability of these two flows when they are subjected to small time-dependent disturbances.

In the opening sections of this paper we shall be concerned with the interaction of small plane disturbances and plane shock waves. The results of both this plane wave investigation and an independent alternative procedure will then be used to show that both configurations are stable.

2. The wave equations. The fundamental equations which define the motion of an ideal compressible fluid are those implying the conservation of momentum, mass, and energy. A convenient form in which they may be presented is the following [in Eq. (3) we have already used the fact that $p=\rho_{0} R T$ ]:

$$
\begin{aligned}
& (\mathrm{V} \cdot \operatorname{grad}) \mathrm{V}+\partial \mathrm{V} / \partial t+\rho_{0}^{-1} \operatorname{grad} p=0, \\
& \mathrm{~V} \cdot \operatorname{grad}\left(\ln \rho_{0}\right)+\partial\left(\ln \rho_{0}\right) / \partial t+\operatorname{div} \mathbf{V}=0, \\
& (\mathrm{~V} \cdot \operatorname{grad}+\partial / \partial t)\left(p / \rho_{0}\right)=0 .
\end{aligned}
$$

Here $\mathbf{V}, p$, and $\rho_{0}$ are respectively, the velocity, pressure, and density, at any point in the field of flow, ${ }^{3}$ and $\gamma$ is the ratio of the specific heats. We shall be interested in the superposition of a small disturbance on a known steady flow. It is therefore convenient to write

$$
\begin{aligned}
\mathrm{V} & =\mathrm{U}_{2}+a_{2}(\operatorname{grad} \varphi+\operatorname{curl} \mathbf{E}), \\
p & =p_{2}(1+\gamma q), \\
\rho_{0} & =\rho_{2}(1+\rho) .
\end{aligned}
$$

*Received Sept. 6, 1947; revised March 1, 1948.

'We imply vanishing viscosity and heat conductivity, and the validity of the ideal gas law, $p=\rho R T$.

${ }^{2} \mathrm{~A}$. Kantrowitz has successfully produced the strong shock solutions.

${ }^{3}$ The subscript zero merely distinguishes the density from the variable without subscript which will soon arise. 
Here, the subscript 2 characterized the state parameters associated with the steady flow downstream of the shock. The disturbance is characterized by the functions, $\varphi$, $q, \rho$, and E. No justification is needed for the foregoing representation of the disturbance velocity except to note that any vector can be expressed in this manner. In fact, we may (and shall) still require without loss of generality that $\operatorname{div} \mathbf{E}$ should vanish identically.

Using now a perturbation process to linearize Eqs. (1), (2), (3), with regard to the disturbance parameters, ${ }^{4}$ we obtain the following linear differential equation

$$
\begin{aligned}
\Delta \varphi-d^{2} \varphi / d t^{2} & =0, \\
d \mathbf{E} / d t & =0, \\
d S / d t & =0 .
\end{aligned}
$$

It is implied in the manipulations which lead to these equations that

$$
q=-d \varphi / d t \text {. }
$$

In Eqs. (7) to (10) (and henceforth) $t$ represents the time multiplied by $a_{2} ; M$ is the Mach number $\left|\mathbf{U}_{2}\right| / a_{2} ; \Delta$ is the Laplace operator; $d / d t=\partial / \partial t+\mathbf{M}_{2} \cdot \operatorname{grad} ;$ and $S=$ $q-\rho$.

It is of interest to interpret these equations from a physical point of view. Equation (7) implies that the function $\varphi$ represents an acoustic wave which propagates according to a conventional wave equation. The vector $\mathbf{E}$ characterizes that part of the velocity vector which implies a non-vanishing vorticity and, according to Eq. (8), drifts with the stream. Finally, $S$, the linearized entropy, also drifts with the stream. The fact that these latter quantities propagate in this manner is, of course, well known [4].

3. The boundary conditions at the shock. In this section we shall be concerned with the formulation of the conditions which must apply at the downstream side of the shock when the steady flow field is disturbed in the immediate neighborhood of the shock. We shall be interested only in those situations where the upstream field remains undisturbed (i.e. either the disturbance originates in the downstream field or sufficient time has elapsed since its formation so that the upstream field is again free from any perturbations). Since, for a stationary shock, the Rankine-Hugoniot conditions $s^{5}$ uniquely determine the state variables on the downstream side in terms of the upstream state variables, no time dependent changes in the field can occur unless we admit oscillations of the shock locus which have a magnitude consistent with that of the disturbance functions. At any time $t$, then, the shock is displaced from its steady state position by an amount ${ }^{6} \psi=\psi(y, z, t)$. In order to determine the downstream state variables we must apply the Rankine-Hugoniot conditions to a shock moving with a velocity $\psi_{t}$ and oriented with regard to its equilibrium configuration at angles $\psi_{y}, \psi_{z}$. Here, we have implied the omission of higher order terms ${ }^{7}$ in $\psi_{y}, \psi_{t}, \cdots$. This linearization is essential if the procedure is to be consistent with that of Sec. 2.

"Details of such a process may be found in [4]. The most naive approach, however, gives the same result as a rigorous analysis.

'See [1], [2], [3].

${ }^{6} \mathrm{Here}$, we choose rectangular coordinate axes as follows: the axis $x$ is normal to the plane of the shock, the plane $z=0$ is normal to both $U_{1}$ and $U_{2}$.

'The angles, of course, are actually $\arctan \psi_{y}, \cdots$. 
Before writing down these jump conditions, the following notation must be introduced $^{8}$

$$
\mathbf{U}_{2}=\mathbf{i} u_{2}+\mathbf{j} v_{2} ; \quad \mathbf{U}_{1}=\mathbf{i} u_{1}+\mathbf{j} v_{1} ;
$$

$u_{1}^{*}, v_{1}^{*}, w_{1}^{*}, u_{2}^{*}, v_{2}^{*}, w_{2}^{*}$, are the upstream and downstream components of velocity in-

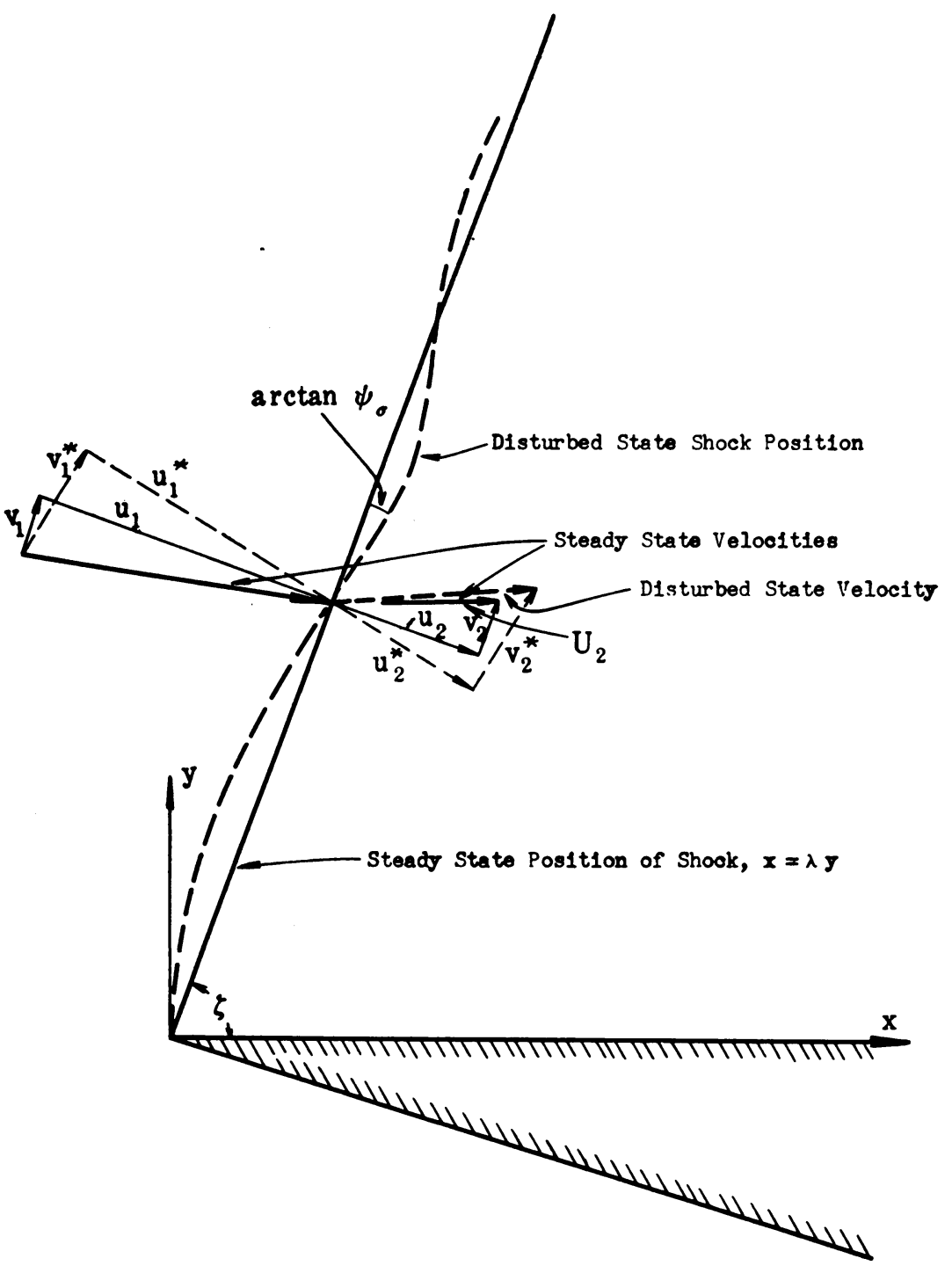

Fig. 1

The coordinate system depicted here is applicable only to the work of Sec. 5. The earlier work utilizes $x=0$ as the shock locus. In this diagram, of course, the $z$ components of velocity are not visible.

${ }_{\mathbf{i}}, \mathbf{j}, \mathbf{k}$ will denote the conventional Cartesian unit vectors. 
stantaneously normal to and tangent to (in planes $z=0$ and $y=0$, respectively) the displaced shock locus; $\delta u_{2}, \delta v_{2}, \delta w_{2}, \delta \rho_{2}, \delta p_{2}$, are the changes in $u_{2}, \cdots, p_{2}$, associated with the shock displacement. From Fig. 1, it is readily seen that (after linearization with regard to $\psi_{y}, \psi_{z}$ )

$$
\begin{array}{ll}
u_{1}^{*}=u_{1}-v_{1} \psi_{\nu}, & v_{1}^{*}=v_{1}+u_{1} \psi_{\nu}, \\
u_{2}^{*}=u_{2}-v_{2} \psi_{\nu}+\delta u_{2}, & v_{2}^{*}=v_{2}+u_{2} \psi_{\nu}+\delta v_{2}, \\
w_{1}^{*}=u_{1} \psi_{2}, & w_{2}^{*}=u_{2} \psi_{z}+\delta w_{2} .
\end{array}
$$

The Rankine-Hugoniot conditions may now be written in the forms

$$
\begin{gathered}
\rho_{1}\left(u_{1}^{*}-\psi_{t}\right)=\left(\rho_{2}+\delta \rho_{2}\right)\left(u_{2}^{*}-\psi_{t}\right), \\
p_{1}+\rho_{1}\left(u_{1}^{*}-\psi_{t}\right)^{2}=p_{2}+\left(\rho_{2}+\delta \rho_{2}\right)\left(u_{2}^{*}-\psi_{t}\right)^{2}, \\
\gamma R\left(T_{1}-T_{2}\right) /(\gamma-1)+\left(u_{1}^{*}-\psi_{t}\right)^{2} / 2 \\
=\frac{\gamma p_{2}}{(\gamma-1) \rho_{2}}\left(\delta p_{2} / p_{2}-\delta \rho_{2} / \rho_{2}\right)+\left(u_{2}^{*}-\psi_{t}\right)^{2} / 2 \\
v_{1}^{*}=v_{2}^{*}, \quad w_{1}^{*}=w_{2}^{*} .
\end{gathered}
$$

If we now substitute Eqs. (11) to (13) into Eqs. (14) to (17), linearize with regard to all disturbance parameters (i.e. $\delta u_{2}, \delta p_{2}, \cdots, \psi_{v}$ ), and utilize the identities ${ }^{\vartheta} \rho_{1} u_{1}=$ $\rho_{2} u_{2}, \cdots$, we obtain

$$
\begin{aligned}
\frac{\delta u_{2}}{a_{2}}= & \frac{a_{2}^{2}+u_{2}^{2}-(\gamma-1) u_{1} u_{2}}{a_{2}^{2}-u_{2}^{2}}\left(1-\rho_{1} / \rho_{2}\right)\left(\psi_{t}+\frac{v_{2}}{a_{2}} \psi_{\nu}\right) \\
\frac{\delta v_{2}}{a_{2}} & =\left(1-\rho_{1} / \rho_{2}\right)\left(u_{1} / a_{2}\right) \psi_{\nu} \\
\frac{\delta w_{2}}{a_{2}}= & \left(1-\rho_{1} / \rho_{2}\right)\left(u_{1} / a_{2}\right) \psi_{z} \\
\frac{\delta p}{\gamma p_{2}}= & -\frac{u_{2}}{a_{2}} \cdot \frac{2 a_{2}^{2}+(\gamma-1)\left(u_{2}^{2}-u_{1} u_{2}\right)}{a_{2}^{2}-u_{2}^{2}} \cdot\left(1-\rho_{1} / \rho_{2}\right)\left(\psi_{t}+\frac{v_{2}}{a_{2}} \psi_{\nu}\right) \\
& \frac{\delta p}{\gamma p_{2}}-\frac{o \rho}{\rho_{2}}=-(\gamma-1) \frac{u_{1}-u_{2}}{a_{2}}\left(1-\rho_{1 /} \rho_{2}\right)\left(\psi_{t}+\frac{v_{2}}{a_{2}} \psi_{\nu}\right)
\end{aligned}
$$

The five quantities on the left sides of these equations must be associated with the values of $\operatorname{grad} \varphi+\operatorname{curl} \mathbf{E},-d \varphi / d t$, and $S$, on the plane $x=0$. In fact, if we now take $\gamma=1.40$, and define $m=u_{2} / a_{2}, n=v_{2} / a_{2}$, and use the fact that ${ }^{10} u_{1} u_{2}=a^{* 2}-$ $(\gamma-1) v_{2}^{2} /(\gamma+1)$, we can write the boundary conditions in the form

${ }^{9}$ These identities, of course, are the steady state Rankine-Hugoniot Conditions.

${ }^{10} \mathrm{Here} a^{*}$ is the critical velocity. 


$$
\begin{aligned}
{[\operatorname{grad} \varphi+\operatorname{curl} \mathbf{E}]_{x=0} } & =\left(1-\rho_{1} / \rho_{2}\right)\left[\mathbf{i} \alpha_{1}\left(\psi_{t}+n \psi_{y}\right)+\mathbf{j} \alpha_{2} \psi_{l y}+\mathbf{k} \alpha_{2} \psi_{z}\right], \\
{[-d \varphi / d t]_{x=0} } & =\left(1-\rho_{1} / \rho_{2}\right) \alpha_{3}\left(\psi_{t}+n \psi_{y}\right), \\
{[S]_{x=0} } & =\left(1-\rho_{1} / \rho_{2}\right) \alpha_{4}\left(\psi_{t}+n \psi_{y}\right),
\end{aligned}
$$

where

$$
\begin{aligned}
& \alpha_{1}=\left(2+4 m^{2}\right) / 3\left(1-m^{2}\right), \\
& \alpha_{2}=\left(5+m^{2}\right) / 6 m, \\
& \alpha_{3}=-m\left(5+m^{2}\right) / 3\left(1-m^{2}\right), \\
& \alpha_{4}=-\left(1-m^{2}\right) / 3 m .
\end{aligned}
$$

4. The interaction of a plane acoustic wave and a shock. Let us now consider the hypothetical situation where the semi-infinite space $x>0$ is unobstructed so that a uniform field exists downstream of the shock located at $x=0$. Furthermore let us consider solutions of Eqs. (7), (8), (9), subject to the boundary conditions (23), (24), (25), which are of the form ${ }^{11}$

$$
\begin{aligned}
\varphi & =A f(t-\xi x-\eta y-\zeta z) \\
\mathbf{E} & =(\mathbf{i} B+\mathbf{j} C+\mathbf{k} D) f(t-\sigma x-\eta y-\zeta z) \\
S & =F f(t-\sigma x-\eta y-\zeta z) \\
\psi & =G f(t-\eta \eta y-\zeta z) .
\end{aligned}
$$

This form implies that the disturbance consists of a plane acoustic wave with wave fronts $\xi x+\eta y+\zeta z=$ const. and of similar vorticity and entropy waves. In order that these waves obey Eqs. (7), (8), (9), one sees by a simple substitution that

and

$$
\xi^{2}+\eta^{2}+\zeta^{2}-(1-m \xi-n \eta)^{2}=0
$$

$$
1-m \sigma-n \eta=0 \text {. }
$$

Here, we have used the notation of the foregoing section in writing $d / d t=\partial / \partial t+$ $m \partial / \partial x+n \partial / \partial y$. The boundary conditions at $x=0$ are given by Eqs. (23). (24), (25), and in this case (supplemented by the condition $\operatorname{div} \mathbf{E} \equiv 0$ ). They take the form

$$
\left[\begin{array}{crrrc}
-\xi & 0 & \zeta & -\eta & -\alpha_{1}(1-n) \\
-\eta & -\zeta & 0 & \sigma & \alpha_{2} \\
-\zeta & \eta & -\sigma & 0 & \alpha_{2} \\
0 & \sigma & \eta & \zeta & 0 \\
1-m-n & 0 & 0 & 0 & \alpha_{3}(1-n \eta)
\end{array}\right]\left[\begin{array}{c}
A \\
B \\
C \\
D \\
G
\end{array}\right]=0
$$

${ }^{11}$ It will turn out that these solutions are certain eigenfunctions. We shall interpret them later. 
and

$$
F=\alpha_{4}(1-n \eta) G .
$$

It is readily shown that this implies $B=0$, and that Eq. (36) can be reduced, with the aid of Eq. (34) and (35), to the form

$$
\left|\begin{array}{ccc}
\mu & 1-\mu^{2} & \frac{4+8 m^{2}}{5+m^{2}} \\
0 & 1 & 1 \\
1 & 0 & -2 m
\end{array}\right|=0
$$

where $\mu=\left[1-\left(\eta^{2}+\zeta^{2}\right)\left(1-m^{2}\right) /(1-n \eta)^{2}\right]^{1 / 2}$.

The solution of Eq. (38) is

$$
\mu=-m \pm\left(1-m^{2}\right) /\left(5+m^{2}\right)^{1 / 2},
$$

and implies that

$$
\xi=(1-n \eta)\left[ \pm\left(5+m^{2}\right)^{-1 / 2}-2 m /\left(1-m^{2}\right)\right] .
$$

Before attempting to interpret this class of solutions, we must establish some supplementary facts. Using the relation ${ }^{12}$

$$
\begin{aligned}
u_{1} u_{2} & =a^{*^{2}}-(\gamma-1) v_{2}^{2} /(\gamma+1), \\
\left(a_{0} / a_{2}\right)^{2} & =1+(\gamma-1)\left(m^{2}+n^{2}\right) / 2,
\end{aligned}
$$

anc

$$
2 a_{0}^{2} /(\bar{\gamma}+1)<u_{1}^{2}+v_{1}^{2}=u_{1}^{2}+v_{2}^{2}<2 a_{0} /(\gamma-1) .
$$

(the first inequality is implied by the second law of thermodynamics) we may conclude that the range of physically possible flows is given by

$$
0 \leq n^{2}<\infty, \quad(\gamma-1) / 2 \gamma<m^{2} \leq 1 .
$$

This in turn implies that the bracket in Eq. (41) is always negative for such flows. Finally Eq. (40) implies that $1-\mu^{2}$ never vanishes or becomes singular; Eq. (39) then implies that $(1-n \eta)^{2}$ never vanishes or becomes singular, and hence $(1-n \eta)$ is always positive. These conclusions require that $\xi$ be always negative [see Eq. (41)] and we have established that no waves of the form given in Eqs. (30) to (33) can be downstream moving waves. That is to say, a negative $\xi$ implies a wave moving in the negative $x$ direction.

The waves implied by the foregoing analysis can now be interpreted in the following manner. A plane acoustic wave with wave fronts $\xi x+\eta y+\zeta z=t$ impinges on the shock at $x=0$ and reflects not as an acoustic wave but as an "entropy wave" $S$ and a "vorticity wave" $\mathbf{E}$. In general, of course, an acoustic wave moving into the shock would reflect as a combination of entropy, vorticity, and acoustic waves, and hence the foregoing solutions are those eigen-solutions for which the amplitude_of the acoustic reflection vanishes.

${ }^{12}$ Here, $a_{0}$ is the stagnation acoustic velocity of the steady downstream field. 
If one wished to consider the general reflection problem, the following procedure would be indicated. Eq. (30) would be replaced by

$$
\varphi=A_{1} f\left(t-\xi_{1} x-\eta y-\zeta z\right)+A_{2} f\left(t-\xi_{2} x-\eta y-\zeta z\right),
$$

where for given $\eta$ and $\zeta$ the values of $\xi_{1}$ and $\xi_{2}$ are the two roots of Eq. (34). In this problem the incident wave is represented by $A_{1} f(t-\cdots-\zeta z)$ and thus $A_{1}$ and the function $f$ are known. The boundary value problem of determining the reflection is treated as before, but now the equation replacing Eq. (36) is non-homogeneous and thus there are not eigenvalues to be found but rather one obtains the coefficients $A_{2} / A_{1}, C / A_{1}$, $\cdots$.

5. The wedge flow stability problem. Let us consider the foregoing field of flow where now, however, we insert a solid boundary along the plane $m y-n x=0$ (i.e. along the direction of the vector $\mathbf{U}_{2}$ ). We are interested in the existence within the region above this plane (i.e. the wedge surface) of oscillations which occur without the aid of external excitation. The boundary conditions which now apply are those already formulated for the boundary $x=0$, the condition that the velocity component normal to the wedge surface vanish at the wedge, and the requirement that the wave be outgoing.

Let us now consider that an acoustic disturbance which vanishes outside of a region $R\left(y_{0}<y<y_{1}\right)$ exists in the field. This disturbance may be considered as being composed of plane waves. ${ }^{13}$ Some of these waves are moving away from the origin. Within a finite time these waves leave the region $R$ and neither they nor their reflections from shock and wedge can return. Any waves moving toward the origin reflect appropriately from wedge and shock and, within a finite time, reach the origin. Here the nature of the combined reflection is not evident. In fact, if the region $R$ is to be disturbed for more than a finite time, the disturbance introduced at the origin must be self-sustaining once it is initiated. It is evident, then, that we must look for an eigenfunction of our problem in a form that defines the behavior near the origin. In the following, we shall not worry about convergence in the large. The solutions considered will be of a type such that the leading terms of the expansions determine the eigenvalue. This will turn out to be such a value that whether the function is defined over the complete region or not, the solutions desired do not exist. We shall first consider those flows for which the down-stream field is supersonic.

It is convenient at this stage to use a coordinate system such that the $x$-axis is rotated about the $z$-axis into the upper wedge surface. Using this rotated coordinate system (see Fig. 1), we may write solutions of Eqs. (7), (8), (9), in the form

$$
\begin{aligned}
\varphi & =f(x, y) \exp \left\{i c\left[t-M x /\left(M^{2}-1\right)\right]\right\} \cos \alpha z, \\
\mathbf{E} & =[\{\mathbf{i} F(y)+\mathrm{j} G(y)\} \sin \alpha z+\mathbf{k} H(y) \cos \alpha z] \exp \left\{i c\left[t-\frac{x}{M}-\frac{\lambda y}{M\left(M^{2}-1\right)}\right]\right\} \\
S & =S(y) \exp \left\{i c\left[t-x / M-\lambda y / M\left(M^{2}-1\right)\right]\right\} \cos \alpha z .
\end{aligned}
$$

Here $f(x, y)$ must satisfy

$$
f_{x x}-f_{y y} /\left(M^{2}-1\right)+k^{2} f=0
$$

${ }^{13}$ Either a discrete set or a continuum of such waves may be needed here. 
where $k^{2}=c^{2} /\left(M^{2}-1\right)^{2}+\alpha^{2} /\left(M^{2}-1\right)$, and $\mathbf{E}$ and $S$ satisfy Eqs. (8), (9) independent of the dependence on $y$ of $F, G, H, S(y)$. We may also write

$$
\psi=g(y) \exp \left\{i c\left[t-M \lambda y /\left(M^{2}-1\right)\right]\right\} \cos \alpha z \text {. }
$$

Finally, defining $\beta^{2}=M^{2}-1, r^{2}=x^{2}-\beta^{2} y^{2}, \theta=\operatorname{arctanh} \beta y / x$, solutions of Eq. (49) may be written in the form,

$$
f_{\nu}(x, y)=J_{\nu}(k r) \cosh \nu \theta .
$$

These functions are such that $f_{y}$ vanishes at $y=0$ and is non-singular at the origin if the admissible values of $\nu$ are governed by $\operatorname{Re}(\nu)>0$. This latter condition will be critical in determining the stability of the flow. Using Eqs. (46) to (48) and (50), the boundary conditions Eqs. (18) to (22) and the condition $\operatorname{div} \mathbf{E}=0$ may now be expressed in the following form.

$$
\begin{aligned}
f_{x}(\lambda y, y)-\frac{i c M}{M^{2}-1} f(\lambda y, y)+H^{\prime}(y)-\alpha G(y) & =A_{1} g(y)+B_{1} g^{\prime}(y), \\
f_{y}(\lambda y, y)+\alpha F(y)+\frac{i c}{M} H(y) & =A_{2} g(y)+B_{2} g^{\prime}(y), \\
-\alpha f(\lambda y, y)-\frac{i c}{M} G(y)-F^{\prime}(y) & =A_{3} g(y)+B_{3} g^{\prime}(y), \\
\frac{i c}{M^{2}-1} f(\lambda y, y)-M f_{x}(\lambda y, y) & =A_{4} g(y)+B_{4} g^{\prime}(y), \\
S(y) & =A_{5} g(y)+B_{5} g^{\prime}(y), \\
\frac{-i c}{M} F(y)+G^{\prime}(y)-\alpha H(y) & =0 .
\end{aligned}
$$

The number $A_{i}, B_{i}$, given here can readily be deduced from Eqs. (18) to (22) and are functions of two parameters ${ }^{14}$ say $m$ and $n$.

Let us now consider the function

$$
f=\sum_{\mu=0}^{\infty} a_{\mu} f_{v+\mu}(x, y)
$$

as defined in Eq. (51). This choice for $f$ and the associated choices of $F(y), \cdots S(y)$ are to satisfy Eqs. (38) to (43). We can anticipate that such functions will be appropriate only for certain eigenvalues $\nu$. Let us assume, for the moment that $\nu \neq 0$. Then we must write

$$
\begin{aligned}
& F(y)=\sum_{\mu=0}^{\infty} b_{\mu} J_{\nu+\mu}(k \xi y), \\
& G(y)=\sum_{\mu=0}^{\infty} c_{\mu} J_{\nu+\mu}(k \xi y),
\end{aligned}
$$

where $\xi=\left(1+\lambda^{2}-M^{2}\right)^{1 / 2}$.

${ }^{14}$ Actually, in Eqs. (18) to (22), they are given as functions of $u_{1}, u_{2}, v_{2}, a_{2} \cdots$, but each of these can be written as $u_{1}=u_{1}(m, n), \cdots$. 
If we now substitute these functions.into Eqs. (52) to (57). and segregate the equalities in the coefficients of $J_{v+\mu}(k \xi y)$ for each $\mu$, we find that the equality in the coefficients of $J_{v-1}(k \xi y)$. constitutes a homogeneous condition for the eigenvalue. The remaining equalities constitute non-homogeneous systems from which the $a_{i}, b_{i}{ }^{\circ}, \cdots$, for $j>0$ may be evaluated.

If one carries out the foregoing process, the characteristic equation for $\nu$ can be written (after algebraic reduction)

$$
\left|\begin{array}{rcc}
\beta \sinh (\nu-1) & \theta_{0} & B_{2} \\
-M \cosh (\nu-1) & \theta_{0} & B_{4}
\end{array}\right|=0,
$$

where $\theta_{0}=\operatorname{arctanh}(\beta / \lambda)$. It is also implied that the lowest order non-vanishing coefficient $b_{n}$ corresponds to $n=1$ whereas the lowest order coefficient in $H$ is $d_{0}$. Thus the boundary condition at $y=0$ can be satisfied only if $H(0)=0$, i.e. only if $\operatorname{Re}(\nu)>0$. It can readily be shown that $B_{2}<0, B_{4}<0, \beta / \lambda<M B_{2} / \beta B_{4}<\lambda / \beta$, for all wedge flows. ${ }^{15}$ This leads to the fact that $\operatorname{Re}(\nu)<0$ for all such flows. It is therefore evident that the type of disturbance defined here does not exist.

Before these considerations can be interpreted as implying the non-existence of eigenfunctions of the type sought (i.e sustained oscillations), one more detail must be supplied. It is quite possible that any such eigenfunction might have a branch point at the origin which was not of the type admitted by the Bessel function expansion. ${ }^{16}$ For example, one might expect that, near the origin, the wave function behaves like $r^{\nu}(\ln r) h(\theta)$. That this is not the case can readily be shown. Without algebraic detail, in fact, the procedure is the following. We consider a solution of the differential equation (7) in the form of an expansion whose leading term is $r^{\nu}(\ln r) h(\theta)$, and define appropriate functions $\mathbf{E}$ and $S$ consistent with this expansion. Then, as with the Bessel function expansion, the equalities among the coefficients of the leading terms which are implied by the boundary conditions lead to a transcendental equation for the eigenvalue $v$. Again, as one can readily verify, $\operatorname{Re}(\nu)$ is negative ${ }^{17}$ for physically possible wedge flows and thus this type of eigenfunction is not admissible. As a matter of fact, it is not even certain that the expansion considered here leads to a function which is defined throughout the field. If not, this consideration is superfluous but in any event an oscillation of this type cannot exist.

At this point we must extrapolate these results and assume that no other type of branch point will lead to admissible solutions. One can readily obtain the general result that for a branch point of type $r^{n} g(r)$, where $g(r)$ is singular at the origin, $R e(n)$ will be negative. Therefore, using this extrapolation (or, if the reader prefers, this conjecture), we conclude that the flow configurations associated with a supersonic downstream field are stable. This conclusion is, of course, based on the fact that no self-excited oscillations ( an exist which vanish identically near the origin (the plane wave results imply this:) ind that no non-singular solutions obey the boundary conditions in the neighborhood of the origin. This, of course, is in agreement with experimental evidence.

${ }^{15}$ See appendix for details.

${ }^{16}$ This possibility was first suggested to the author by S. S. Shu.

${ }^{17}$ In fact, the same $\nu_{i}$ are associated with this solution as were found for the preceding expansion. 
The subsonic case is more easily disposed of. The definition $\beta^{2}=1-M^{2}, r^{2}=$ $x^{2}+\beta^{2} y^{2}, \theta=\operatorname{arc} \tan \beta y / x$, will lead to a set of differential equations and their solutions which are analogous to those found by the corresponding procedure in the supersonic case. Again, a set of Bessel functions multiplied this time by trigonometric functions in $\theta$ will form a product series solution. However, in order that the waves be outgoing in the subsonic field, we must now use the Hankel functions $H_{\nu}^{(2)}$. These are singular for all $\nu$, of course, and again this type of series is not admissible. Therefore, with an extrapolation of the same type we used before, we must conclude that the subsonic field is also stable.

In view of the somewhat unusual nature of our results, it seems worth while to summarize briefly without recourse to the physical picture. In the supersonic (subsonic) case, once we have demanded solutions of Eqs. (7) in the form $f(x, y) e^{i c t}$, we have a hyperbolic (elliptic) second order partial differential equation with constant coefficients. The homogeneous boundary conditions require $\varphi_{n}=0$ on $\theta=0, L\left(\varphi, \varphi_{n}\right)=0$ on $\theta=\theta_{0}$, an outgoing wave, and the overriding condition that $\varphi$ be non-singular at the origin. Here, $L$ represents a linear combination of the argument functions and their derivatives with regard to $r, \varphi_{n}$ is the normal derivative of $\varphi$. Actually, the first three conditions given here are sufficient to find a unique set of eigenfunctions in either case. The satisfaction of the "overriding" condition (or the lack thereof) then determines the admissibility of these eigenfunctions and in our case is not satisfied. Thus the tentatively chosen functions are not suitable and hence no sustained oscillations exist.

6. Some general comments. It seems of interest now to speculate on the causes of the fact that the weak shock is usually observed in experimental work. Each of the flow configurations will be altered (as indicated in the fore-going) by any externally generated disturbances. In the supersonic case, such disturbances can get into the field only through the boundary layer and even then must propagate downstream within their Mach cone. On the other hand, disturbances originating at the trailing end of the finite wedge may go upstream in a subsonic stream and the forced oscillations of the strong shock may be sufficiently great to "push it over" into the other flow. Essentially the same point of view may be stated in the form: A disturbance in the weak shock field has a very small probability of getting near the origin where the overall behavior is determined. In the strong shock field, large disturbances are continually fed towards the origin, and unless such disturbances can be minimized sufficiently, the transition to the weak shock flow will occur.

7. A remark on a previous investigation. In a recent paper [5], J. I. Levinson has treated this same stability problem also using the small time-dependent-perturbation theory. He, however, considers a sustained excitation proceeding from the upstream field toward the downstream field. Although it is true that random upstream disturbances might initiate downstream perturbations, the proper investigation must not admit sustained excitation but must consider only the eigenfunction perturbations. We must therefore consider the physical argument as presented in that paper as incorrect for the problems which are usually of physical interest. Furthermore, in the boundary conditions at the shock, the author utilizes the fact that the shock is wavy but neglects the fact that it is moving. That is, he omits the terms corresponding to $\psi_{t}$ in our Eqs. (18) to (22). Hence, the actual calculations cannot even be used to find the response of the downstream field to an upstream excitation. His conclusions, which imply in- 
stability of all strong shocks and many weak shocks, are consistently contradicted by experimental evidence.*

\section{APPENDIX}

The inequalities which imply $\operatorname{Re}(\nu)<0$ may be developed as follows

$$
\begin{aligned}
B_{2} & =\frac{5+m^{2}}{6 M}-\frac{\left(2+4 m^{2}\right) n^{2}}{3\left(1-m^{2}\right) M}, \\
B_{4} & =-m n \frac{5+m^{2}}{3\left(1-m^{2}\right)}, \\
\lambda & =n / m, \\
\beta^{2} & =n^{2}+m^{2}-1=M^{2}-1 .
\end{aligned}
$$

We shall use the inequalities

$$
1>m^{2}>1 / 7, \quad n^{2}>1-m^{2} .
$$

We have, then,

$$
\begin{gathered}
-B_{2}=+\frac{\left(4+8 m^{2}\right) n^{2}-\left(5+m^{2}\right)\left(1-m^{2}\right)}{6 M\left(1-m^{2}\right)}>\frac{\left(7 m^{2}-1\right)\left(1-m^{2}\right)}{6 M\left(1-m^{2}\right)}>0 \\
\left(M B_{2} / \lambda B_{4}\right)-\frac{\beta}{\lambda}=\frac{\beta}{\lambda} \cdot \frac{\left(4+8 m^{2}\right) n^{2}-\left(5+m^{2}\right)\left(1-m^{2}\right)-2 \beta^{2} m^{2}\left(5+m^{2}\right)}{2 \beta^{2}\left(5+m^{2}\right) m^{2}} \\
>\frac{\beta}{\lambda} \cdot \frac{\left(7 m^{2}-1\right)\left(1-m^{2}\right)}{2 \beta^{2} n^{2}\left(5+m^{2}\right)}>0 .
\end{gathered}
$$

Hence $M B_{2} / \lambda B_{4}>\beta / \lambda$.

Furthermore

$$
\begin{aligned}
\frac{M B_{2}}{\lambda B_{4}}-\frac{\lambda}{\beta} & =\frac{\lambda}{\beta} \cdot \frac{\left(4+8 m^{2}\right) n^{2}-\left(5+m^{2}\right)\left(1-m^{2}\right)-2 n^{2}\left(5+m^{2}\right)}{2\left(5+m^{2}\right) n^{2}} \\
& =\frac{\lambda}{\beta} \cdot \frac{-\left(6 n^{2}+m^{2}+5\right)\left(1-m^{2}\right)}{2 n^{2}\left(5+m^{2}\right)}<0
\end{aligned}
$$

and the inequality is completed.

We may now define $\left(-M B_{2}\right)-\sinh \alpha$ and $\left(-\beta B_{4}\right)=\cosh \alpha$, so that Eq. (59) reduces to

$$
\sinh \left[(\nu-1) \theta_{0}+\alpha\right]=0
$$

or

$$
\nu \theta_{0}=\theta_{0}-\alpha .
$$

*Note added in proof: Certain discussions have occurred while this paper was in proof which indicate that the analysis of the foregoing problem is not complete. Although no eigenfunctions exist which are exponential in time, we must still investigate how an arbitrary initial disturbance grows with time. This problem has been solved for certain special cases and a continued treatment of this stability question will appear as Part II of this paper. 
However, we have shown that $\tanh \alpha>\tanh \theta_{0}$, so for $\tanh \alpha<1, \alpha>\theta_{0}$ and $\nu$ is negative. For $\tanh \alpha=Q>1$, we have $\alpha=1 / 2 \ln (1+Q) /(1-Q)$ and $\operatorname{Re}(\alpha)=1 / 2$ $\ln (1+Q) /(Q-1)=\operatorname{arctanh} 1 / Q$.

Since we have shown $Q^{-1}>\beta / \lambda$ we again have $\operatorname{Re}(\nu)<0$.

\section{BIBLIOGRAPHY}

[1] G. I. Taylor and J. W. Maccoll, The mechanics of compressible fluids, in Durand, Aerodynamic theory, Vol. 3, J. Springer, Berlin, (1934).

[2] H. W. Emmons, Shock waves in aerodynamics, J. Aero. Sci. 12, 188-194 (1945).

[3] J. M. Burgers, On the interaction of shock waves and sound waves, Nederland. Ak. Wetensch 49, 273-281 (1946).

[4] G. F. Carrier and F. D. Carlson, On the propagation of small disturbances in a moning compressible fluid, Q. Appl. Math. 4, 1-12 (1946).

[5] J. I. Levinson, L'étude de la stabilité des courants supersoniques de gaz en relation avec une double solution de la théorie des ondes de choc, Prikl. Mat. i. Mekh. 9, 151-170 (1945). 